Алгебра и анализ

Tом 22 (2010), № 1
St Petersburg Math. J.

Vol. 22 (2011), No. 1, Pages 55-59

S 1061-0022(2010)01132-4

Article electronically published on November 16, 2010

\title{
THE CLOSURE OF THE HARDY SPACE IN THE BLOCH NORM
}

\author{
N. M. GALÁN AND A. NICOLAU \\ Dedicated to V. P. Havin \\ on the occasion of his 75th birthday
}

\begin{abstract}
A description of the closure in the Bloch norm of the Bloch functions that are in a Hardy space is given. The result uses the classical estimates for the Lusin area function.
\end{abstract}

\section{§1. INTRODUCTION}

This paper is devoted to the description of the closure in the Bloch norm of the space $\mathrm{H}^{p} \cap$ Bloch. First, we recall some definitions.

For $0<p<\infty$, the Hardy space $\mathrm{H}^{p}$ is the space of analytic functions $f$ in the unit disk such that

$$
\|f\|_{\mathrm{H}^{p}}^{p}=\sup _{0<r<1} \int_{0}^{2 \pi}\left|f\left(r e^{i \theta}\right)\right|^{p} d \theta<+\infty .
$$

As usual, $\mathrm{H}^{\infty}$ is the space of bounded analytic functions in the unit disk.

We also recall the definition of the space BMO of functions with bounded mean oscillation. Let $u$ be an integrable function on the unit circle $\mathbb{T}$, and let $u_{I}$ denote the mean of $u$ over the $\operatorname{arc} I \subset \mathbb{T}$, that is,

$$
u_{I}=\frac{1}{|I|} \int_{I} u(\xi)|d \xi| .
$$

Here $|d \xi|$ is the normalized Lebesgue measure on $\mathbb{T}$. The function $u$ is in BMO if

$$
\|u\|_{\mathrm{BMO}}=\sup \frac{1}{|I|} \int_{I}\left|u(\xi)-u_{I}\right||d \xi|<+\infty,
$$

where the supremum is taken over all $\operatorname{arcs} I \subset \mathbb{T}$. An analytic function $f$ in the unit disk $\mathbb{D}$ is in the space BMOA if it is the Poisson extension to the disk of a function in BMO.

Finally, recall that a function $f$ is in the Bloch space, denoted by Bloch, if $f$ is analytic in $\mathbb{D}$ and

$$
\|f\|_{\text {Bloch }}=\sup _{z \in \mathbb{D}}\left(1-|z|^{2}\right)\left|f^{\prime}(z)\right|<\infty .
$$

It is well known that $\mathrm{H}^{\infty} \subsetneq \mathrm{BMOA} \subsetneq$ Bloch $\cap \mathrm{H}^{p}$ for any $p<\infty$. Therefore, it is natural to ask for a description of the closure of the spaces $\mathrm{H}^{\infty}, \mathrm{BMOA}$, and $\mathrm{H}^{p} \cap$ Bloch in the Bloch space.

Garnett and Jones gave a description of the closure of $\mathrm{L}^{\infty}$ in the BMO norm, and also of $\mathrm{H}^{\infty}$ in BMOA, based on the John-Nirenberg inequality [6], stating that a function

2010 Mathematics Subject Classification. Primary 30H30.

Key words and phrases. Bloch norm, bounded mean oscillation, Lusin area function.

Supported in part by grants MTM2008-00145 and 2009SGR420. 
$u \in \mathrm{L}^{1}(\mathbb{T})$ is in BMO if and only if there exists $\varepsilon>0$ and a constant $C>0$ such that for any $\operatorname{arc} I \subset \mathbb{T}$ and any $\lambda>0$ we have

$$
\left|\left\{\xi \in I:\left|u(\xi)-u_{I}\right|>\lambda\right\}\right| \leq C e^{-\lambda / \varepsilon}|I| .
$$

In [3, Garnett and Jones showed that a function $u$ is in the closure of $\mathrm{L}^{\infty}$ in the BMO norm if and only if for any $\varepsilon>0$ there exists a constant $C=C(\varepsilon)>0$ such that the inequality above is satisfied.

A characterization of the closure of BMOA in the Bloch norm is also due to P. Jones. Given a function $f \in$ Bloch and $\varepsilon>0$, define

$$
\Omega_{\varepsilon}(f)=\left\{z \in \mathbb{D}:\left(1-|z|^{2}\right)\left|f^{\prime}(z)\right| \geq \varepsilon\right\} .
$$

Then $f$ is in the closure of BMOA in the Bloch norm if and only if for every $\varepsilon>0$ there exists a constant $C=C(\varepsilon)>0$ such that

$$
\int_{Q \cap \Omega_{\varepsilon}(f)} \frac{d A(z)}{1-|z|^{2}} \leq C \ell(Q)
$$

for any Carleson square $Q$ of the form

$$
Q=\left\{r e^{i \theta}: 0<1-r<\ell(Q),\left|\theta-\theta_{0}\right|<\ell(Q)\right\}, \quad 0 \leq \theta_{0}<2 \pi .
$$

In [5], Ghatage and Zheng gave a proof of this result and they attributed it to P. Jones. Our main purpose in this paper is to adapt this proof to give a description of the Bloch functions that can be approximated in the Bloch norm by functions in Bloch $\cap \mathrm{H}^{p}$. This result is stated in Theorem 1 below.

We start with some notation. Given a set $\Omega \subseteq \mathbb{D}$, let $A_{h}(\Omega)$ be the hyperbolic area of $\Omega$, that is,

$$
A_{h}(\Omega)=\int_{\Omega} \frac{d A(z)}{\left(1-|z|^{2}\right)^{2}} .
$$

Also, for fixed $M>1$ and for $\xi \in \mathbb{T}$, let $\Gamma(\xi)=\{z \in \mathbb{D}:|z-\xi|<M(1-|z|)\}$ be the Stolz angle with vertex at $\xi$. Our main result is as follows.

Theorem 1. Let $1<p<\infty$, and let $f$ be a function in the Bloch space. Then $f$ is in the closure in the Bloch norm of Bloch $\cap \mathrm{H}^{p}$ if and only if for any $\varepsilon>0$ the function $A_{h}^{1 / 2}\left(\Omega_{\varepsilon}(f) \cap \Gamma(\xi)\right)$ is in $\mathrm{L}^{p}(\mathbb{T})$.

In the case where $p=2$, this condition can be written in a more pleasant way. The Fubini theorem allows us to restate our result as follows: A function $f \in$ Bloch is in the closure of $\mathrm{H}^{2} \cap$ Bloch if and only if for any $\varepsilon>0$ we have

$$
\int_{\Omega_{\varepsilon}(f)} \frac{d A(z)}{1-|z|^{2}}<\infty
$$

Notice that the condition in Peter Jones' result is the conformally invariant version of the previous one.

The necessity in our result follows easily from well-known estimates on the Lusin area function. For $f \in \mathrm{H}^{p}$, the area function of $f$ at the point $\xi \in \mathbb{T}$ is defined as

$$
A(f)(\xi)=\left(\int_{\Gamma(\xi)}\left|f^{\prime}(z)\right|^{2} d A(z)\right)^{1 / 2} .
$$

The following characterization of $\mathrm{H}^{p}$ spaces in terms of the area function will be used (see [7] and [8, p. 224]).

Theorem A. Let $0<p<+\infty$, and let $f$ be an analytic function in the unit disk. Then $f \in \mathrm{H}^{p}$ if and only if $A(f) \in \mathrm{L}^{p}(\mathbb{T})$. Moreover, the norms $\|f\|_{\mathrm{H}^{p}}$ and $\|A(f)\|_{\mathrm{L}^{p}}$ are comparable. 
The proof of the sufficiency in Theorem 1 is more difficult. Here we proceed as in the proof of Jones' theorem given in [5]. If $f \in$ Bloch and $f(0)=f^{\prime}(0)=0$, then for any $z \in \mathbb{D}$ we have the following reproducing formula:

$$
f(z)=\int_{\mathbb{D}} \frac{\left(1-|w|^{2}\right) f^{\prime}(w)}{(1-\bar{w} z)^{2} \bar{w}} d A(w)
$$

see 2]. We shall show that the integral over $\mathbb{D} \backslash \Omega_{\varepsilon}(f)$ has small Bloch norm, so that we shall need to check that the integral over $\Omega_{\varepsilon}(f)$ is in $\mathrm{H}^{p}$. This will be accomplished by a duality argument, again with the use of estimates of the Lusin area function.

It is important to remark that our arguments do not apply to the case of $p=\infty$, because Theorem $\AA$ fails for $p=\infty$. The problem of describing the closure of $\mathrm{H}^{\infty}$ in the Bloch norm, first stated in [1], remains an open problem.

\section{§2. Proof of Theorem 1}

Fix $1<p<\infty$. First, we show necessity. So, let $f$ be a function in the closure of the space $\mathbf{H}^{p} \cap$ Bloch. Then, given $\varepsilon>0$, we can find $g \in \mathbf{H}^{p} \cap$ Bloch such that $\|f-g\|_{\text {Bloch }} \leq \varepsilon / 2$. Since $\Omega_{\varepsilon}(f) \subseteq \Omega_{\varepsilon / 2}(g)$, for any $\xi \in \mathbb{T}$ we have

$$
A_{h}\left(\Omega_{\varepsilon}(f) \cap \Gamma(\xi)\right) \leq \int_{\Omega_{\varepsilon / 2}(g) \cap \Gamma(\xi)} \frac{d A(z)}{\left(1-|z|^{2}\right)^{2}} \leq \int_{\Gamma(\xi)} \frac{4}{\varepsilon^{2}}\left|g^{\prime}(z)\right|^{2} d A(z) .
$$

Since $g \in \mathrm{H}^{p}$, Theorem $\mathrm{A}$ shows that its area function is in $\mathrm{L}^{p}(\mathbb{T})$, and we deduce that $A_{h}^{1 / 2}\left(\Omega_{\varepsilon}(f) \cap \Gamma(\xi)\right) \in \mathrm{L}^{p}(\mathbb{T})$.

Conversely, fix $p$ with $1<p<\infty$, and let $f$ be a function in the Bloch space such that for any $\varepsilon>0$ the function $A_{h}^{1 / 2}\left(\Omega_{\varepsilon}(f) \cap \Gamma(\xi)\right)$, as a function of $\xi \in \mathbb{T}$, is in $L^{p}(\mathbb{T})$. Fix $\varepsilon>0$. We are going to construct a function $f_{1} \in \mathrm{H}^{p} \cap$ Bloch such that $\left\|f-f_{1}\right\|_{\text {Bloch }}<\varepsilon$. We proceed as in [5]. We may assume that $f(0)=f^{\prime}(0)=0$ and $\|f\|_{\text {Bloch }}=1$. In [2] it was proved that

$$
f(z)=\int_{\mathbb{D}} \frac{\left(1-|w|^{2}\right) f^{\prime}(w)}{(1-\bar{w} z)^{2} \bar{w}} d A(w)
$$

for all $z \in \mathbb{D}$. We denote $\Omega_{\varepsilon}=\Omega_{\varepsilon}(f)$, split the integral into two parts, and define

$$
f_{1}(z)=\int_{\Omega_{\varepsilon}} \frac{\left(1-|w|^{2}\right) f^{\prime}(w)}{(1-\bar{w} z)^{2} \bar{w}} d A(w)
$$

and

$$
f_{2}(z)=\int_{\mathbb{D} \backslash \Omega_{\varepsilon}} \frac{\left(1-|w|^{2}\right) f^{\prime}(w)}{(1-\bar{w} z)^{2} \bar{w}} d A(w),
$$

so that $f=f_{1}+f_{2}$. Since

$$
\left|f_{2}^{\prime}(z)\right| \leq 2 \varepsilon \int_{\mathbb{D}} \frac{d A(z)}{|1-\bar{w} z|^{3}}, \quad z \in \mathbb{D},
$$

we deduce that $\left\|f_{2}\right\|_{\text {Bloch }} \leq C \varepsilon$. Hence, we only need to show that $f_{1}$ is in $\mathbf{H}^{p}$. This will be accomplished as in [5] by a duality argument.

Without loss of generality we may assume that $f_{1}(0)=0$. Let $\overline{\mathrm{H}^{q}}$ be the space of antianalytic functions in the unit disk such that $\bar{g} \in \mathrm{H}^{q}$. Consider the operator

$$
T(g)=\int_{\mathbb{D}} \overline{\bar{g}^{\prime}(z)} f_{1}^{\prime}(z) \log \frac{1}{|z|} d A(z), \quad g \in \overline{\mathrm{H}^{q}} .
$$

The argument below will show that there exists a fixed constant $C=C(\varepsilon)>0$ such that $|T(g)| \leq C\|\bar{g}\|_{\mathrm{H}^{q}}$ for any $g \in \overline{\mathrm{H}^{q}}$. Once this inequality is established, we shall see that $T$ 
gives rise to a bounded linear functional on $\overline{\mathrm{H}^{q}}$. Then, by duality, there exists $F \in \mathrm{H}^{p}$ such that

$$
T(g)=\int_{\mathbb{T}} F\left(e^{i \theta}\right) g\left(e^{i \theta}\right) d \theta
$$

for any $g \in \overline{\mathrm{H}^{q}}$. Here $d \theta$ is the normalized angular measure. Since $T(1)=0$, we have $F(0)=0$. By the Littlewood-Paley integral formula (see [4, p. 228]), we have

$$
T(g)=\int_{\mathbb{D}} F^{\prime}(z) \overline{\bar{g}^{\prime}(z)} \log \frac{1}{|z|} d A(z)
$$

for all $g \in \overline{\mathrm{H}^{q}}$. Then $F=f_{1}$, which shows that $f_{1} \in \mathrm{H}^{p}$. So, we need to check that there exists a constant $C=C(\varepsilon)>0$ such that

$$
\left|\int_{\mathbb{D}} \overline{\bar{g}^{\prime}(z)} f_{1}^{\prime}(z) \log \frac{1}{|z|} d A(z)\right| \leq C\|\bar{g}\|_{\mathrm{H}^{q}}, \quad g \in \overline{\mathrm{H}^{q}} .
$$

First, observe that the Fubini theorem gives

$$
T(g)=2 \int_{\Omega_{\varepsilon}}\left(1-|w|^{2}\right) f^{\prime}(w) \int_{\mathbb{D}} \frac{\overline{\bar{g}^{\prime}(z)}}{(1-\bar{w} z)^{3}} \log \frac{1}{|z|} d A(z) d A(w) .
$$

Next, we show that the integral

$$
\int_{\mathbb{D}} \frac{\bar{g}^{\prime}(z)}{(1-w \bar{z})^{3}} \log \frac{1}{|z|} d A(z)
$$

is essentially the derivative of a certain function in $\mathrm{H}^{q}$. To check this, we fix $w \in$ $\mathbb{D} \backslash\{0\}$ and apply the Littlewood-Paley integral formula to the functions $\bar{g}(z)$ and $\left(2 w(1-w \bar{z})^{2}\right)^{-1}$ to obtain

$$
\int_{\mathbb{D}} \frac{\bar{g}^{\prime}(z)}{(1-w \bar{z})^{3}} \log \frac{1}{|z|} d A(z)=\int_{0}^{2 \pi} \frac{\bar{g}\left(e^{i \theta}\right)}{2 w\left(1-w e^{-i \theta}\right)^{2}} d \theta-\frac{\bar{g}(0)}{2 w} .
$$

Now we can use the variable $\xi=e^{i \theta}$, and by the Cauchy integral formula we can express the right-hand side of the last identity as

$$
\frac{1}{2 w i} \int_{\mathbb{T}} \frac{(\bar{g}(\xi)-\bar{g}(0)) \xi}{(\xi-w)^{2}} d \xi=\frac{1}{2 w} h^{\prime}(w),
$$

where $h(w)=(\bar{g}(w)-\bar{g}(0)) w$. So, finally we obtain

$$
T(g)=\int_{\mathbb{D}}\left(1-|w|^{2}\right) f^{\prime}(w) \chi_{\Omega_{\varepsilon}}(w) \frac{1}{\bar{w}} \overline{h^{\prime}(w)} d A(w) .
$$

Since $f^{\prime}(0)=0$, there exists $C_{1}=C_{1}(\varepsilon)$ such that $\Omega_{\varepsilon} \subset\left\{w \in \mathbb{D}:|w| \geq C_{1}\right\}$. Therefore, taking modules, we deduce that

$$
\begin{aligned}
|T(g)| & \leq \frac{1}{C_{1}} \int_{\mathbb{D}}\left(1-|w|^{2}\right)\left|f^{\prime}(w)\right|\left|h^{\prime}(w)\right| \chi_{\Omega_{\varepsilon}}(w) d A(w) \\
& \leq \frac{1}{C_{1}} \int_{\mathbb{D}}\left|h^{\prime}(w)\right| \chi_{\Omega_{\varepsilon}}(w) d A(w) .
\end{aligned}
$$

By the Fubini theorem, we deduce that there exists a constant $C_{2}>0$ such that

$$
|T(g)| \leq C_{2} \int_{\mathbb{T}} \int_{\Gamma(\xi) \cap \Omega_{\varepsilon}} \frac{\left|h^{\prime}(w)\right|}{1-|w|^{2}} d A(w)|d \xi| .
$$


Applying the Cauchy-Schwarz inequality to the inner integral, we get

$$
\begin{aligned}
|T(g)| & \leq C_{2} \int_{\mathbb{T}}\left(\int_{\Omega_{\varepsilon} \cap \Gamma(\xi)} \frac{d A(w)}{\left(1-|w|^{2}\right)^{2}}\right)^{\frac{1}{2}}\left(\int_{\Gamma(\xi)}\left|h^{\prime}(w)\right|^{2} d A(w)\right)^{\frac{1}{2}}|d \xi| \\
& =C_{2} \int_{\mathbb{T}} A_{h}^{1 / 2}\left(\Omega_{\varepsilon} \cap \Gamma(\xi)\right) A(h)(\xi)|d \xi| .
\end{aligned}
$$

By the hypothesis that $A_{h}^{1 / 2}\left(\Omega_{\varepsilon} \cap \Gamma(\xi)\right)$ is in $\mathrm{L}^{p}(\mathbb{T})$ and by Theorem $\mathrm{A}$, the area function $A(h)$ is in $\mathrm{L}^{q}(\mathbb{T})$; so using finally the Hölder inequality and the hypothesis, we deduce that

$$
\begin{aligned}
|T(g)| & \leq C_{2}\left\|A_{h}^{1 / 2}\left(\Omega_{\varepsilon} \cap \Gamma(\xi)\right)\right\|_{L^{p}(\mathbb{T})}\|A(h)\|_{L^{q}(\mathbb{T})} \\
& \leq C_{3}\|h\|_{L^{q}(\mathbb{T})} \leq 2 C_{3}\|g\|_{L^{q}(\mathbb{T})}
\end{aligned}
$$

for any $g \in \overline{\mathrm{H}^{q}}$. This inequality gives (2), so that $T(g)$ determines a bounded linear functional on $\overline{\mathrm{H}^{q}}$. Hence, Theorem 1 is proved.

\section{REFERENCES}

[1] J. M. Anderson, J. Clunie, and Ch. Pommerenke, On Bloch functions and normal functions, J. Reine Angew. Math. 270 (1974), 12-37. MR0361090 (50:13536)

[2] S. Axler and K. H. Zhu, Boundary behavior of derivatives of analytic functions, Michigan Math. J. 39 (1992), no. 1, 129-143. MR1137894 (93e:30073)

[3] J. B. Garnett and P. W. Jones, The distance in BMO to $L^{\infty}$, Ann. of Math. (2) 108 (1978), no. 2, 373-393. MR0506992 (80h:46037)

[4] J. B. Garnett, Bounded analytic functions, Grad. Texts in Math., vol. 236, Springer, New York, 2007. MR2261424 (2007e:30049)

[5] P. G. Ghatage and D. C. Zheng, Analytic functions of bounded mean oscillation and the Bloch space, Integral Equations Operator Theory 17 (1993), no. 4, 501-515. MR.1243993 (94i:30031)

[6] F. John and L. Nirenberg, On functions of bounded mean oscillation, Comm. Pure Appl. Math. 14 (1961), 415-426. MR0131498 (24:A1348)

[7] J. Marcinkiewicz and A. Zygmund, A theorem of Lusin. I, Duke Math. J. 4 (1938), no. 3, 473-485. MR.1546069

[8] E. M. Stein, Singular integrals and differentiability properties of functions, Princeton Math. Ser., No. 30, Princeton Univ. Press, Princeton, NJ, 1970. MR0290095 (44:7280)

Departament de Matemàtiques, Universitat Autònoma de Barcelona, 08193 Bellaterra, BARCELONA, SPAIN

E-mail address: nachomg@mat.uab.es

Departament de Matemàtiques, Universitat Autònoma de Barcelona, 08193 Bellaterra, BARCELONA, Spain

E-mail address: artur@mat.uab.cat

Received 17/SEP/2009

Originally published in English 